\title{
Systematic study of proton-neutron pairing correlations in the nuclear shell model
}

\author{
Y. Lei \\ Department of Physics, Shanghai Jiao Tong University, Shanghai 200240, China, Bartol Research Institute and Department of Physics and \\ Astronomy, University of Delaware, Newark, Delaware 19716, USA
}

S. Pittel

Bartol Research Institute and Department of Physics and Astronomy, University of Delaware, Newark, Delaware 19716, USA

N. Sandulescu

Institute of Physics and Nuclear Engineering, 76900 Bucharest, Romania

A. Poves

Departamento de Fisica Teorica and IFT UAM/CSIC, Universidad Autonoma de Madrid, E-28049 Madrid, Spain

B. Thakur

Bartol Research Institute and Department of Physics and Astronomy, University of Delaware, Newark, Delaware 19716, USA and LONI Institute and Center for Computational Technology, Louisiana State University, Baton Rouge, Lousisiana 70803-4001, USA

Y. M. Zhao

Department of Physics, Shanghai Jiao Tong University, Shanghai 200240, China

(Received 14 July 2011; revised manuscript received 19 September 2011; published 20 October 2011)

\begin{abstract}
A shell-model study of proton-neutron pairing in $2 p 1 f$ shell nuclei using a parametrized Hamiltonian that includes deformation and spin-orbit effects as well as isoscalar and isovector pairing is reported. By working in a shell-model framework we are able to assess the role of the various modes of proton-neutron pairing in the presence of nuclear deformation without violating symmetries. Results are presented for ${ }^{44} \mathrm{Ti},{ }^{45} \mathrm{Ti},{ }^{46} \mathrm{Ti},{ }^{46} \mathrm{~V}$, and ${ }^{48} \mathrm{Cr}$ to assess how proton-neutron pair correlations emerge under different scenarios. We also study how the presence of a one-body spin-obit interaction affects the contribution of the various pairing modes.
\end{abstract}

DOI: 10.1103/PhysRevC.84.044318

\section{INTRODUCTION}

It is generally believed that proton-neutron $(p n)$ pairing is important in nuclei with roughly equal numbers of neutrons and protons [1]. The standard technique for treating these correlations is through the Bardeen-Cooper-Schrieffer (BCS) or Hartree-Fock-Bogolyubov (HFB) approximation, generalized to include the $p n$ pairing field in addition to the neutron-neutron $(n n)$ and proton-proton $(p p)$ pairing fields [1]. Questions arise, however, as to whether these methods can adequately represent the physics of the competing modes of pair correlations, without full restoration of symmetries [2].

Important insight into this issue was achieved recently in the context of exactly solvable models that include these different pairing modes. Analysis of the $\mathrm{SO}(8)$ model [3], in which isoscalar and isovector pairing act in either a single active orbital or a series of degenerate orbitals, suggests that isospin restoration or equivalently quartet correlations are extremely important, especially near $N=Z$ [2]. More recent studies, carried out for models involving nondegenerate orbitals [4], reinforce earlier conclusions as to where isoscalar pairing correlations should be most important [5,6]. Furthermore, they make possible the description of deformation, as is critical for systems with $N \approx Z$, by treating the nondegenerate orbitals as Nilsson-like. However, it is still not possible to restore certain symmetries within these models, for example, rotational symmetry.
PACS number(s): 21.60.Cs, 21.60.Fw, 02.30.Ik, 21.30.Fe

As a consequence, there still remain many open issues concerning the role of the different possible modes of pairing in $N \approx Z$ nuclei. In this work, we report a systematic study of pairing correlations in the context of the nuclear shell model, whereby deformation can be readily included and symmetries maintained throughout. In this way, we are able to address many of the open issues on the role of the various pairing modes in the presence of nuclear deformation.

An outline of the paper is as follows. In Sec. II, we briefly describe our model and then in Sec. III describe selected results. Finally, in Sec. IV we summarize the key conclusions of the paper.

\section{OUR MODEL}

To address in a systematic way the role of pairing correlations in the presence of nuclear deformation, we consider neutrons and protons restricted to the orbitals of the $2 p 1 f$ shell outside a doubly magic ${ }^{40} \mathrm{Ca}$ core and interacting via a schematic Hamiltonian

$$
H=\chi\left(Q \cdot Q+a P^{\dagger} \cdot P+b S^{\dagger} \cdot S+\alpha \sum_{i} \vec{l}_{i} \cdot \vec{s}_{i}\right),
$$

where $Q=Q_{n}+Q_{p}$ is the mass quadrupole operator, $P^{\dagger}$ creates a correlated $L=0, S=1, J=1, T=0$ pair, and $S^{\dagger}$ 
creates a correlated $L=0, S=0, J=0, T=1$ pair. The first term in the Hamiltonian produces rotational collective motion, whereas the second and third terms are the isoscalar and isovector pairing interactions, respectively, whose matrix elements can be found in Ref. [7]. The last term is the one-body part of the spin-orbit interaction, which splits the $j=l \pm 1 / 2$ levels with a given $l$.

We carry out shell-model calculations systematically as a function of the various strength parameters. We begin with pure SU(3) rotational motion [8] associated with the $Q \cdot Q$ interaction and then gradually ramp up the various $\mathrm{SU}(3)$-breaking terms to assess how they affect the rotational properties. This includes the isocalar and isovector pairing interactions and the spin-orbit term.

We first consider the nucleus ${ }^{44} \mathrm{Ti}$, with $N_{n}=N_{p}=2$, and then systematically increase $N_{n}$ and $N_{p}$ to study the role of the number of active neutrons and protons (e.g., whether there is an excess of one over the other and whether the nucleus is even-even, odd-mass, or odd-odd). The nuclei we have treated $\operatorname{are}^{44} \mathrm{Ti}\left(N_{n}=N_{p}=2\right),{ }^{45} \mathrm{Ti}\left(N_{n}=2, N_{p}=3\right),{ }^{46} \mathrm{Ti}\left(N_{n}=2\right.$, $\left.N_{p}=4\right),{ }^{46} \mathrm{~V}\left(N_{n}=3, N_{p}=3\right)$, and ${ }^{48} \mathrm{Cr}\left(N_{n}=4, N_{p}=4\right)$. Some of the observables we have studied are (1) the energies and associated $\mathrm{BE}(2)$ values of the lowest rotational band, (2) the number of isovector $S^{\dagger}$ pairs, and (3) the number of isoscalar $P^{\dagger}$ pairs. In the following, we present selected results that derive from these calculations.

\section{RESULTS}

\section{A. An optimal Hamiltonian}

Before turning to our results for specific nuclei, we first ask whether the Hamiltonian (1) has sufficient flexibility to realistically describe the nuclei under investigation. Without making an effort toward an absolute fit, we note that the choice $\chi=-0.05 \mathrm{MeV}, a=b=12$, and $\alpha=20$ gives an acceptable fit to the spectra of all the nuclei we have considered.

We first show in Fig. 1 its prediction for ${ }^{42} \mathrm{Sc}$, in comparison with the experimental spectrum. Other than its inability to reproduce the low-lying $J^{\pi}=7^{+}, T=0$ state, the optimal

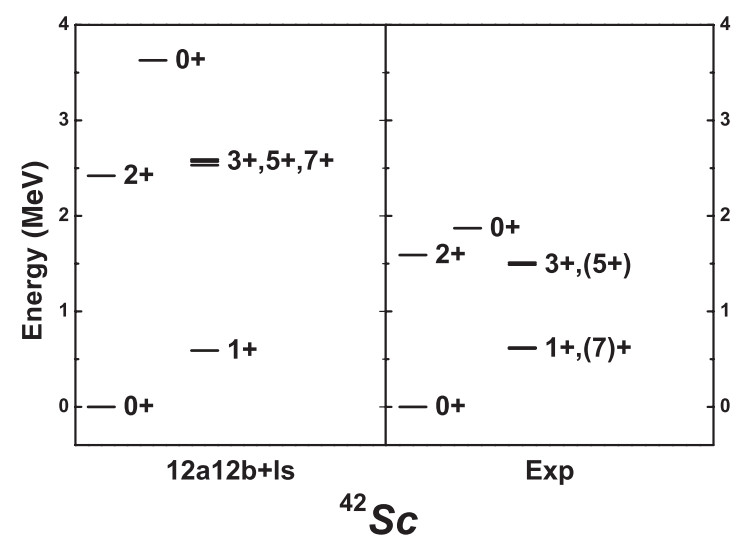

FIG. 1. Comparison of experimental spectra for ${ }^{42} \mathrm{Sc}$ with the calculated spectra obtained using the optimal Hamiltonian described in the text. All energies are in $\mathrm{MeV}$.

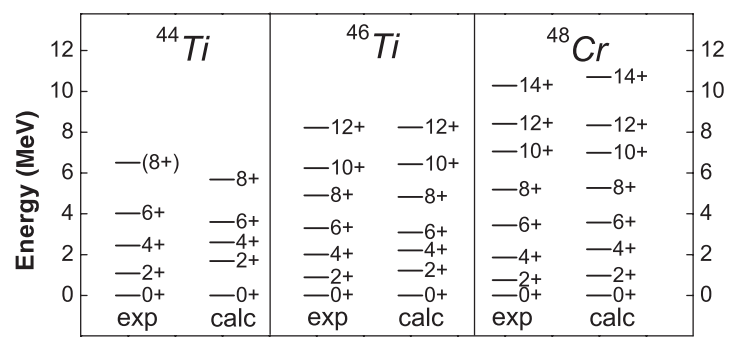

FIG. 2. Comparison of experimental spectra for ${ }^{44} \mathrm{Ti}$, ${ }^{46} \mathrm{Ti}$, and ${ }^{48} \mathrm{Cr}$ with the calculated spectra obtained using the optimal Hamiltonian described in the text. All energies are in $\mathrm{MeV}$.

Hamiltonian does an acceptable job in qualitatively describing the features of the low-energy spectrum. The lack of an acceptable description of the $7^{+}$state reflects the fact that our optimal Hamiltonian is missing the strong attraction between $f_{7 / 2}$ nucleons in the stretch configuration.

In Fig. 2, we show its predictions for ${ }^{44} \mathrm{Ti}$, ${ }^{46} \mathrm{Ti}$, and ${ }^{48} \mathrm{Cr}$. As can be seen, the nonrotational character of ${ }^{44} \mathrm{Ti}$ at low angular momenta is reproduced by our calculations, as are the highly rotational patterns seen experimentally for the heavier nuclei. As we will see later, even the experimentally observed backbend in ${ }^{48} \mathrm{Cr}$ is acceptably reproduced with this Hamiltonian. We refer to the choice $a=b$ in the optimal Hamiltonian as the $\mathrm{SU}$ (4) choice, from the dynamical symmetry that derives from this choice of parameters in the $\mathrm{SO}(8)$ model.

\section{B. ${ }^{44} \mathrm{Ti}$}

The first nucleus we discuss is ${ }^{44} \mathrm{Ti}$, with two active neutrons and two active protons. In Fig. 3, we show the calculated energy splittings $E(I)-E(I-2)$ associated with the ground-state band as a function of the strength parameters $a$ and $b$ that define the isoscalar and isovector pairing interactions, respectively. For these calculations we assumed a quadrupole strength of $\chi=-0.05 \mathrm{MeV}$ and no spin-orbit interaction. As expected, in the absence of a spin-orbit splitting the isoscalar and isovector pairing interactions have precisely the same effect on the properties of the ground-state rotational band. The same conclusion derives when we consider the effect of isoscalar and isovector pairing on other observable properties when no
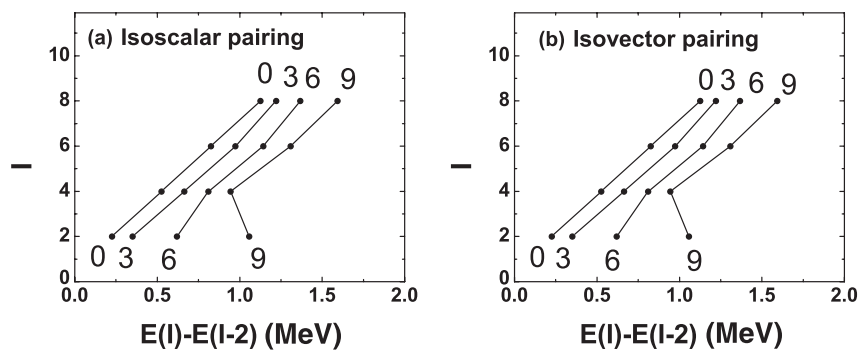

FIG. 3. Calculated energy splittings $E(I)-E(I-2)$ (in $\mathrm{MeV}$ ) in the ground band of ${ }^{44} \mathrm{Ti}$ as a function of the strength of the (a) isoscalar pairing interaction and the (b) isovector pairing interaction, with no spin-orbit splitting. The strengths of the respective pairing interactions are shown at the ends of the lines, as they are elsewhere in the paper. 

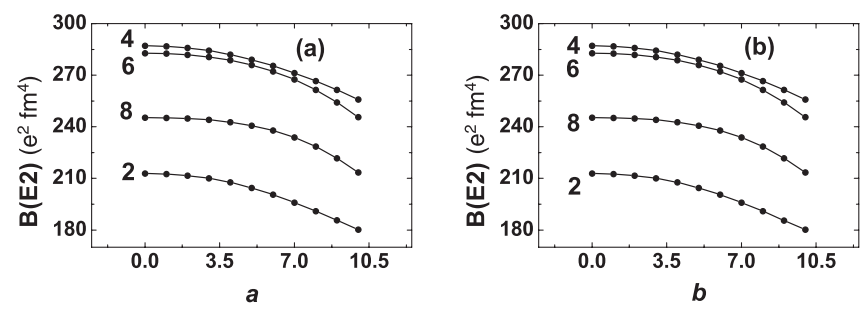

FIG. 4. Calculated $E 2$ transition matrix elements $B(E 2, I \rightarrow$ $I-2)$ in the ground band of ${ }^{44} \mathrm{Ti}$ as a function of the strength of the (a) isoscalar pairing interaction and of the (b) isovector pairing interaction, with no spin-orbit splitting. The angular momentum $I$ of the initial state appears to the left of each line.

spin-orbit splitting is included. As an example, we show in Fig. 4 results for the $\mathrm{BE}(2)$ values connecting the states in the ground band, again as a function separately of the isovector and isoscalar pairing strengths.

We next show in Fig. 5 the same results as in Fig. 2, but now in the presence of our realistic spin-orbit strength $\alpha=20$. Here we see that the effects of isoscalar and isovector pairing are very different. In the presence of realistic single-particle energies, isovector pairing produces a much more rapid loss of rotational collectivity than isoscalar pairing.

Next we consider the relative effect of the spin-orbit interaction on isovector and isoscalar pairing properties in the optimal SU(4) limit, where both modes of pairing contribute with the same strength. This is addressed in Fig. 6, where we show the average number of $S^{\dagger}$ and $P^{\dagger}$ pairs in the ground band as a function of the strength of the spin-orbit force. These are determined by considering $\left\langle S^{\dagger} \cdot S\right\rangle$ and $\left\langle P^{\dagger} \cdot P\right\rangle$ and scaling them with respect to the results that would derive from pure $T=0$ and $T=1$ pairing Hamiltonians (for a system of two pairs in an $\Omega=10$ shell), respectively. While the number of isovector pairs does not change substantially with increasing spin-orbit strength, the isoscalar pair number is reduced dramatically, especially for the lower angular momentum states of the band. We conclude, therefore, that the spin-orbit interaction suppresses isoscalar pairing, already at $N=Z$. The mechanism whereby this suppression takes place was discussed recently in Ref. [9].

Finally, in Fig. 7 we show the spectrum of ${ }^{44} \mathrm{Ti}$ that derives solely from turning on a strong spin-orbit force (i.e., with no pairing present). We see that the spectrum is still highly rotational, despite the fact that the resulting single-particle
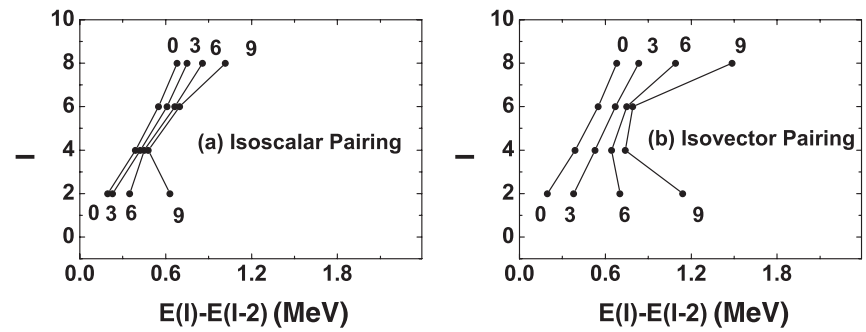

FIG. 5. Calculated energy splittings $E(I)-E(I-2)$ in $\mathrm{MeV}$ in the ground band of ${ }^{44} \mathrm{Ti}$ as a function of the strength of the (a) isoscalar pairing interaction and of the (b) isovector pairing interaction, with the optimal spin-orbit term present.
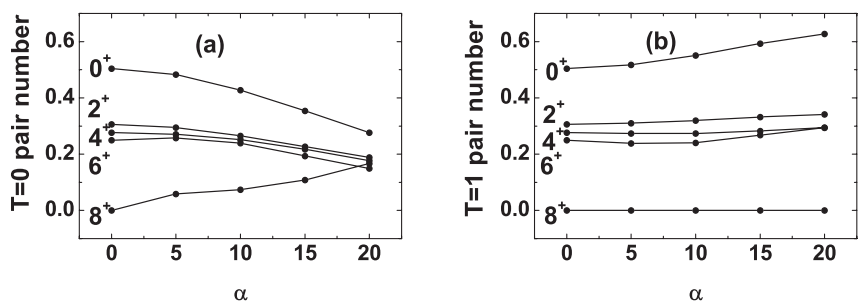

FIG. 6. The number of (a) isocalar $P^{\dagger}$ pairs and (b) isovector $S^{\dagger}$ pairs in ${ }^{44} \mathrm{Ti}$ as a function of the strength of the spin-orbit interaction $\alpha$. All other Hamiltonian parameters are the optimal values.

energies are no longer SU(3)-like. To obtain the physical spectrum with a nonrotational character, it is thus essential to have pairing. It has been traditionally thought that it is the non-SU(3) order of the single-particle levels that is responsible for the nonrotational character seen in the experimental spectrum [10], a conclusion that is not supported by our results. It is pairing that is responsible for the nonrotational character of ${ }^{44} \mathrm{Ti}$. This point was already suggested in Fig. 3, where we we saw that for the physical pairing strengths a highly nonrotational spectrum near the ground band emerged even in the absence of a spin-orbit splitting. Further understanding of this conclusion follows from the important role of quasi-SU(3) [11] in producing deformation. Even though the spin-orbit interaction breaks the SU(3) symmetry, it leaves quasi-SU(3) symmetry approximately preserved.

\section{C. ${ }^{45} \mathrm{Ti}$}

Next we consider the odd-mass nucleus ${ }^{45} \mathrm{Ti}$, with one additional neutron relative to ${ }^{44} \mathrm{Ti}$. For notational purposes, we divide the results according to whether $I-1 / 2$ is odd (referring to this as the odd group) or whether it is even (the even group). This reflects the fact that states within the ground-state band decay by strong $E 2$ transitions within their own groups.

In Figs. 8 and 9, we present results for the calculated energy splittings of the ground band in the odd and even groups, respectively, as a function of pure isoscalar (panel a) and pure

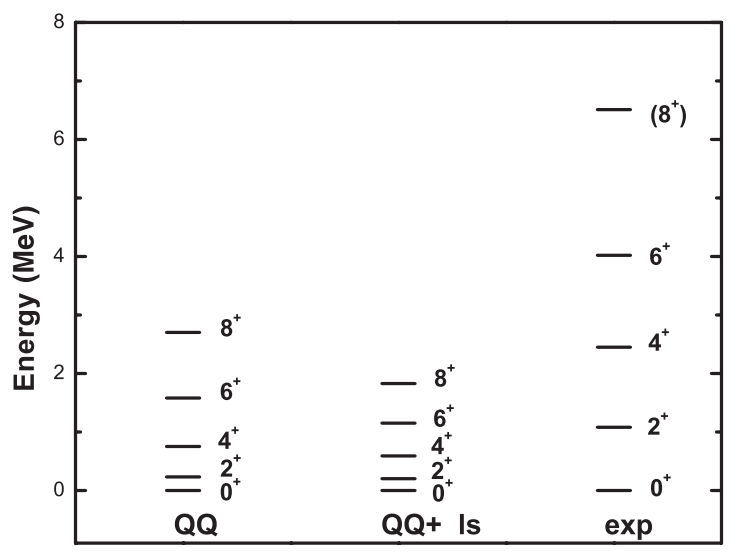

FIG. 7. Comparison of the experimental spectrum of ${ }^{44} \mathrm{Ti}$ with those obtained using a pure $Q \cdot Q$ interaction and both a $Q \cdot Q$ interaction and a spin-orbit term. 

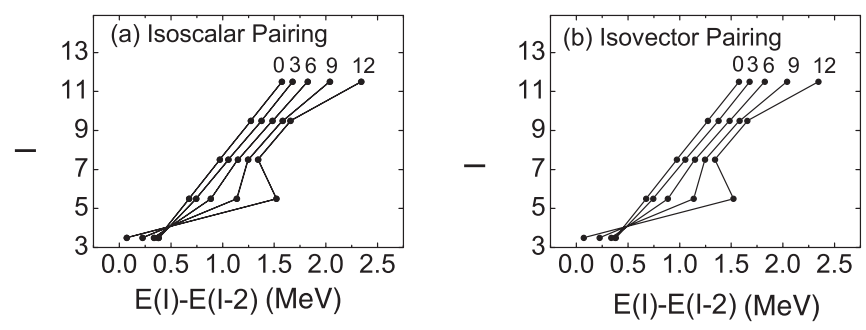

FIG. 8. Calculated energy splittings $E_{I}-E_{I-2}$ in $\mathrm{MeV}$ within the odd group of levels (see text) of the ground-state band of ${ }^{45} \mathrm{Ti}$ as a function of the strength of the (a) isoscalar pairing interaction and of the (b) isovector pairing interaction, with no spin-orbit term present.

isovector (panel b) pairing, in both cases with no spin-orbit force present. The results suggest that blocking due to an odd neutron does not affect the symmetry between isoscalar and isovector pairing when there is no spin-orbit force.

In Figs. 10 and 11, we show the corresponding results in the presence of a spin-orbit force, with the optimal strength $\alpha=20$. Now isoscalar pairing is suppressed and there is a much more dramatic effect of isovector pairing.

\section{D. ${ }^{46} \mathrm{Ti}$}

Next we turn to ${ }^{46} \mathrm{Ti}$ with two excess neutrons present. Here too we compare the effect of the isoscalar and isovector pairing interactions on deformation, showing the results in Fig. 12 with no spin-orbit term present. Here the effect of isoscalar pairing is strongly suppressed relative to isovector pairing, suggesting that even without a spin-orbit term isoscalar pairing is very strongly focused on those nuclei with $N=Z$ with a slight excess being sufficient to suppress this pairing mode.

\section{E. ${ }^{46} \mathrm{~V}$}

We next consider ${ }^{46} \mathrm{~V}$, an odd-odd $N=Z$ nucleus. The $T=1$ states of ${ }^{46} \mathrm{~V}$ are, of course, precisely the same as those already considered in ${ }^{46} \mathrm{Ti}$.

We first address in Fig. 13 the manner whereby the symmetry between isocalar and isovector pairing in the absence of a spin-orbit force is reflected in ${ }^{46} \mathrm{~V}$. In the absence of isoscalar and isovector pairing, the $J=1^{+}$state and the $J=0^{+}$state form a degenerate ground-state doublet.
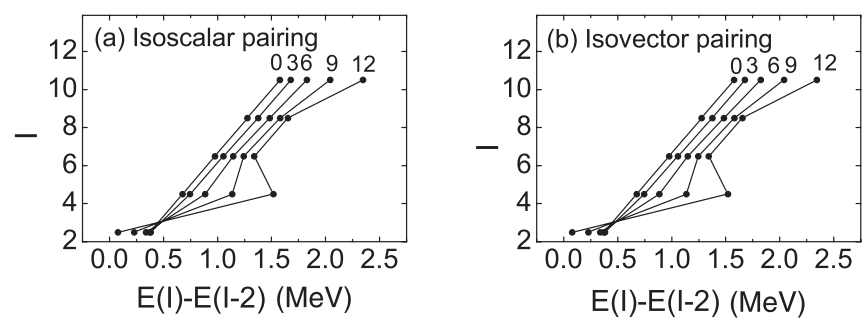

FIG. 9. Calculated energy splittings $E_{I}-E_{I-2}$ within the even group of levels (see text) of the ground-state band of ${ }^{45} \mathrm{Ti}$ as a function of the strength of the isoscalar pairing interaction (left panel) and of the isovector pairing interaction (right panel), with no spin-orbit term present.
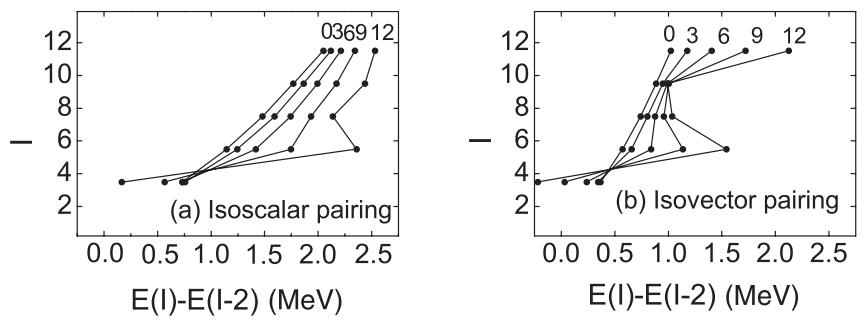

FIG. 10. Calculated energy splittings $E(I)-E(I-2)$ in $\mathrm{MeV}$ within the odd group of levels (see text) of the ground-state band of ${ }^{45} \mathrm{Ti}$ as a function of the strength of the (a) isoscalar pairing interaction and of the (b) isovector pairing interaction, with the optimal spin-orbit term present.

When only isoscalar pairing is turned on (panel a), the $J=1^{+}$ state is pushed down below the $J=0^{+}$state. When only isovector pairing is turned on (panel b) the reverse happens and the $J=0^{+}$is pushed down and becomes the ground state. In the SU(4) limit (panel c) with equal isovector and isocalar pairing strengths, the degeneracy reappears.

In Fig. 14, we show what happens in the presence of the physical spin-orbit interaction, for equal isovector and isoscalar pairing. Now the degeneracy is broken and the $0^{+}$state emerges as the ground state, as in the experiment. The experimental splitting is $1.23 \mathrm{MeV}$, whereas our optimal Hamiltonian produces a splitting of $1.05 \mathrm{MeV}$.

We should note that the first excited state in ${ }^{46} \mathrm{~V}$ experimentally is found to be a $3^{+}$state, at $801 \mathrm{keV}$. In our calculations the lowest $3^{+}$state occurs at significantly higher energy, at $1.89 \mathrm{MeV}$. This may be related to the schematic nature of our Hamiltonian.

\section{F. ${ }^{48} \mathrm{Cr}$}

Lastly, we turn to ${ }^{48} \mathrm{Cr}$, which again has $N=Z$, but now with two quartet structures present. Here we assume as our starting point both the optimal quadrupole-quadrupole force and the optimal one-body spin-orbit force and then ramp up the two pairing strengths from zero to their optimal values. The results are illustrated in Fig. 15, for scenarios in which we separately include isoscalar pairing, isovector pairing, and $\mathrm{SU}(4)$ pairing with equal strengths.
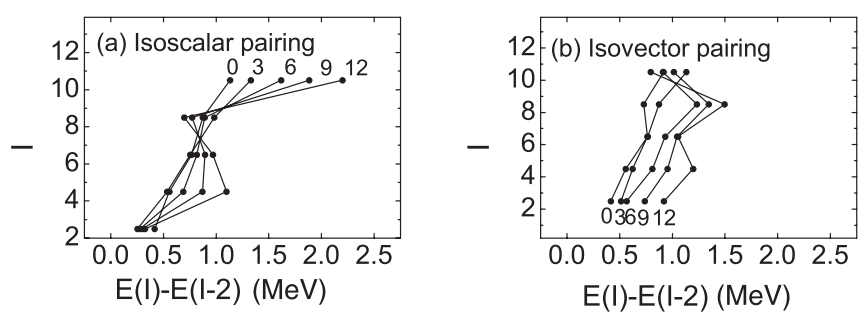

FIG. 11. Calculated energy splittings $E(I)-E(I-2)$ in $\mathrm{MeV}$ within the even group of levels (see text) of the ground-state band of ${ }^{45} \mathrm{Ti}$ as a function of the strength of the (a) isoscalar pairing interaction and of the (b) isovector pairing interaction, with the optimal spin-orbit term present. 

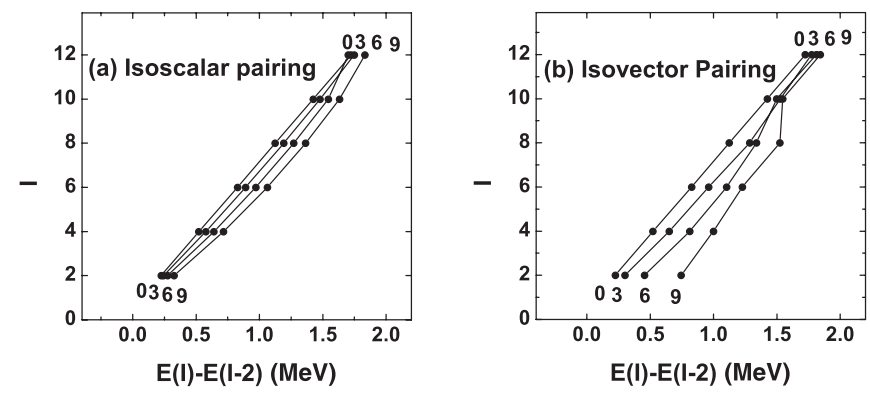

FIG. 12. Calculated energy splittings $E(I)-E(I-2)$ in $\mathrm{MeV}$ in the ground band of ${ }^{46} \mathrm{Ti}$ as a function of the strength of the (a) isoscalar pairing interaction and of the (b) isovector pairing interaction, with no spin-orbit term present.

As a reminder, the experimental spectrum for ${ }^{48} \mathrm{Cr}$ shows a backbend near $I=12$, which as noted earlier is reproduced by our optimal Hamiltonian. The results of Fig. 15 make clear that (a) the backbend cannot be reproduced with pure isoscalar pairing, but requires isovector pairing as well, and (b) there is no significant difference between the results obtained with pure isovector pairing and $\mathrm{SU}(4)$ pairing.

The backbend in ${ }^{48} \mathrm{Cr}$ was discussed extensively in the context of a shell-model study with a fully realistic Hamiltonian in Ref. [7], where it was first shown to derive from isovector pairing. Our results are in agreement with that earlier conclusion. To see these points more clearly, we show in Fig. 16 the numbers of isovector $S^{\dagger}$ and isoscalar $P^{\dagger}$ pairs as a function of angular momentum for the optimal Hamiltonian. As in our treatment of ${ }^{44} \mathrm{Ti}$ (see Fig. 6), the pair numbers are obtained by evaluating $\left\langle S^{\dagger} \cdot S\right\rangle$ and $\left\langle P^{\dagger} \cdot P\right\rangle$ and scaling them with respect to the results that would derive from pure $T=1$ and $T=0$ pairing Hamiltonians, respectively. [Now, however, the analysis is carried out for a system of four pairs in an $\Omega=10$ shell.] As in Ref. [7], the contribution of isovector pairing in the $J=0^{+}$ground state is much larger than the contribution of isoscalar pairing. As the system cranks to higher angular momenta, the isovector pairing contribution
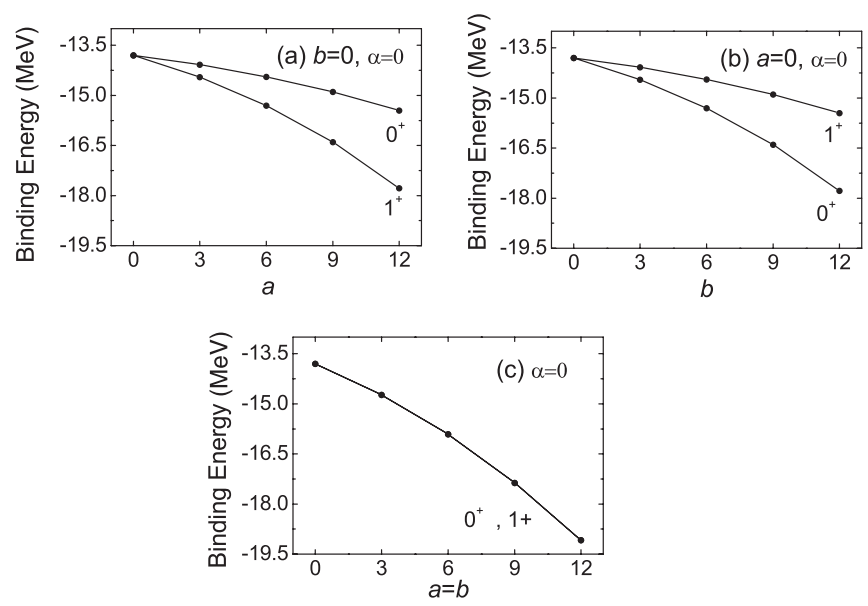

FIG. 13. Calculated energies in $\mathrm{MeV}$ of the lowest $J^{\pi}=0^{+}$and $J^{\pi}=1^{+}$states of ${ }^{46} \mathrm{~V}$ with no spin-orbit term present. Panel (a) shows the results of pure isoscalar pairing, panel (b) shows the results of pure isovector pairing, and panel (c) shows the results of SU(4) pairing.

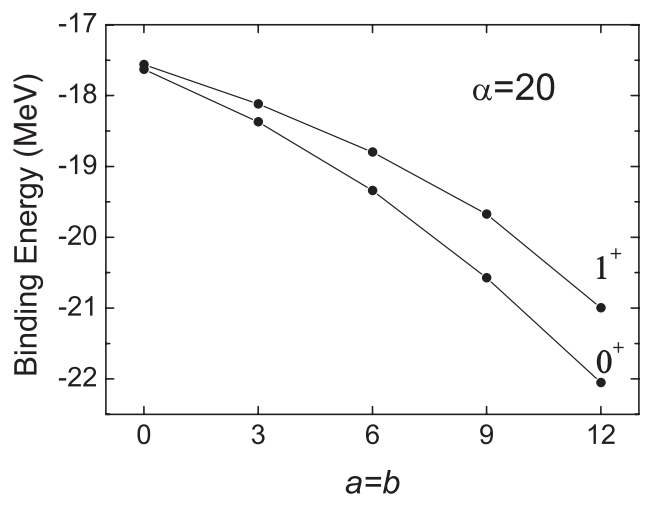

FIG. 14. Calculated energies in $\mathrm{MeV}$ of the lowest $J^{\pi}=0^{+}$and $J^{\pi}=1^{+}$states of ${ }^{46} \mathrm{~V}$ as a function of the equal strength of isoscalar and isovector pairing, with the optimal spin-orbit term $(\alpha=20)$ present.

falls off with angular momentum very rapidly eventually arriving at a magnitude roughly comparable with the isoscalar pairing contribution at roughly $J^{\pi}=10^{+}$. As the angular momentum increases even further we see a fairly substantial increase in the isovector pairing contribution at $J^{\pi}=12^{+}$, which according to Fig. 15 is where the backbend becomes prominent. After the backbend, both isoscalar and isovector pairing contributions decrease to near zero as alignment is achieved.

We have also studied the properties of the lowest excited (yrare) band that emerges from the same calculation, a $K=2^{+}$ band. The energies of this band are illustrated in Fig. 17, together with those of the ground (yrast) band. From this figure, we conclude that the backbend in ${ }^{48} \mathrm{Cr}$ does not derive from level crossing.

In Fig. 18, we show the number of isocalar and isovector pairs in the excited yrare band, to be compared with the results for the yrast band of Fig. 16. In the backbend region, the
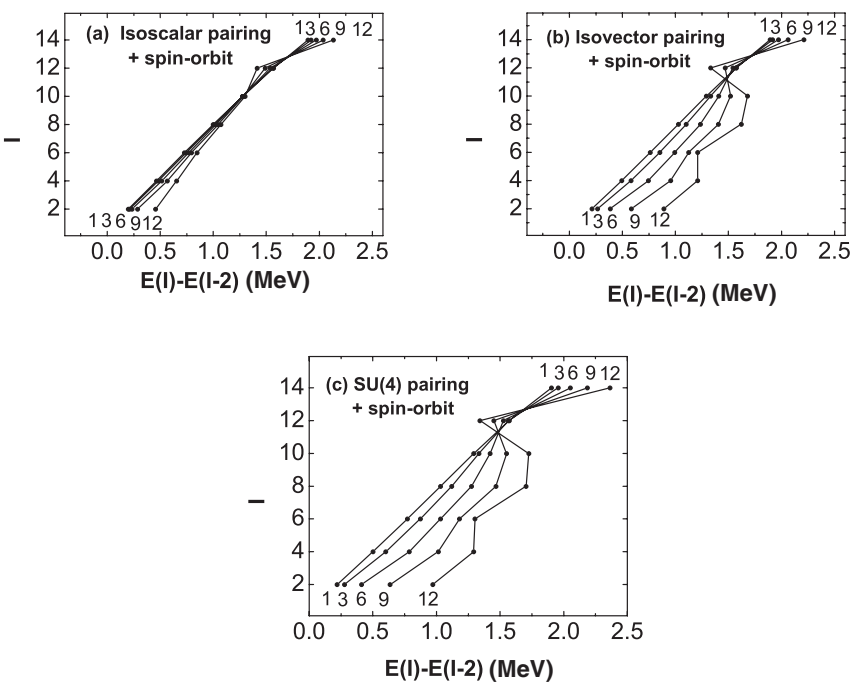

FIG. 15. Calculated energy splittings $E(I)-E(I-2)$ in $\mathrm{MeV}$ in the ${ }^{48} \mathrm{Cr}$ ground band, for (a) isovector, (b) isoscalar, and (c) SU(4) pairing, respectively, as described in the text. The optimal spin-orbit term is included. 


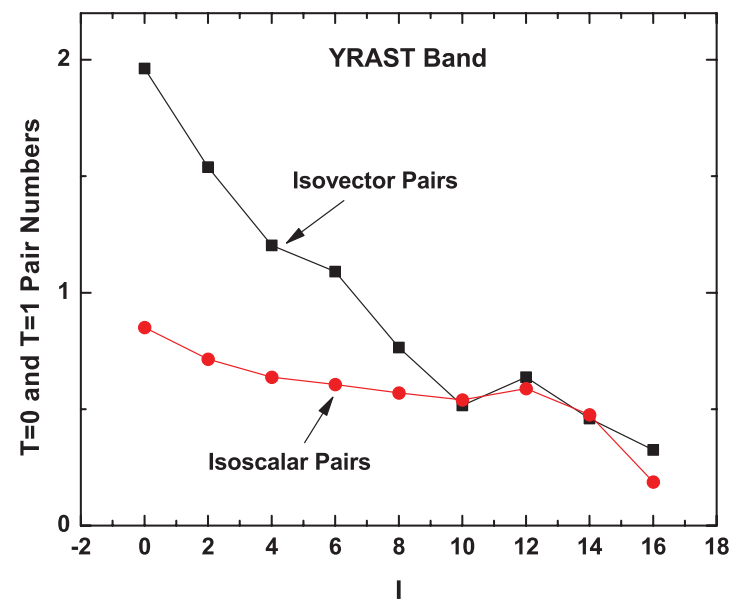

FIG. 16. (Color online) Calculated numbers of isovector $S^{\dagger}$ pairs and isoscalar $P^{\dagger}$ pairs in the ground (yrast) band of ${ }^{48} \mathrm{Cr}$ for the optimal values of the Hamiltonian parameters.

numbers of isoscalar and isovector pairs are found to behave differently in the two bands. Whereas the numbers of isoscalar and isovector pairs are roughly the same in the yrast band (Fig. 16), there are substantially fewer isoscalar pairs than isovector pairs in the yrare band (Fig. 18). We believe that this is an interesting observation worthy of further study.

\section{SUMMARY AND CONCLUDING REMARKS}

In this work, we have reported a systematic shell-model study of proton-neutron pairing in $2 p 1 f$ shell nuclei using a parametrized Hamiltonian that includes deformation and spinorbit effects as well as both isoscalar and isovector pairings. By working in a shell-model framework we are able to assess the role of the various pairing modes in the presence of nuclear deformation without violating symmetries.

We first showed that our parametrized Hamiltonian has enough flexibility to provide a reasonable description of the evolution of nuclear structure properties in this region. We then

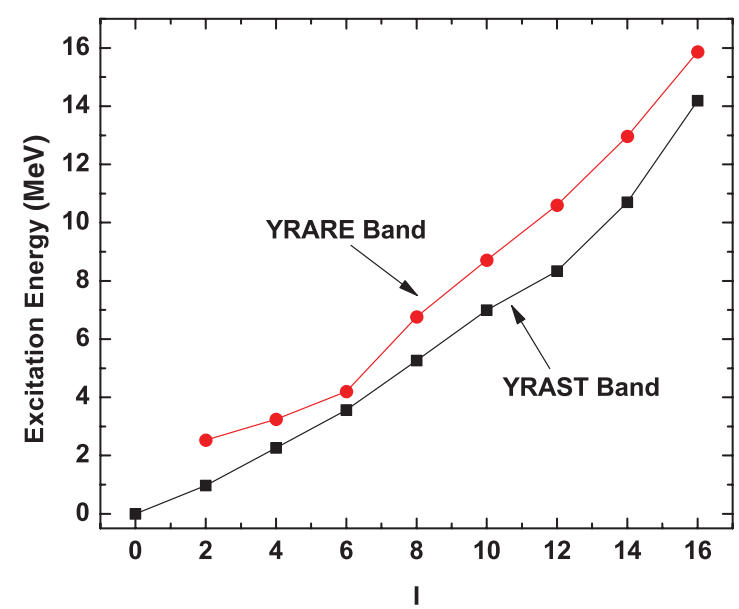

FIG. 17. (Color online) Calculated excitation energies of the ground (yrast) band and the first excited (yrare) band in ${ }^{48} \mathrm{Cr}$ for the optimal values of the Hamiltonian parameters.

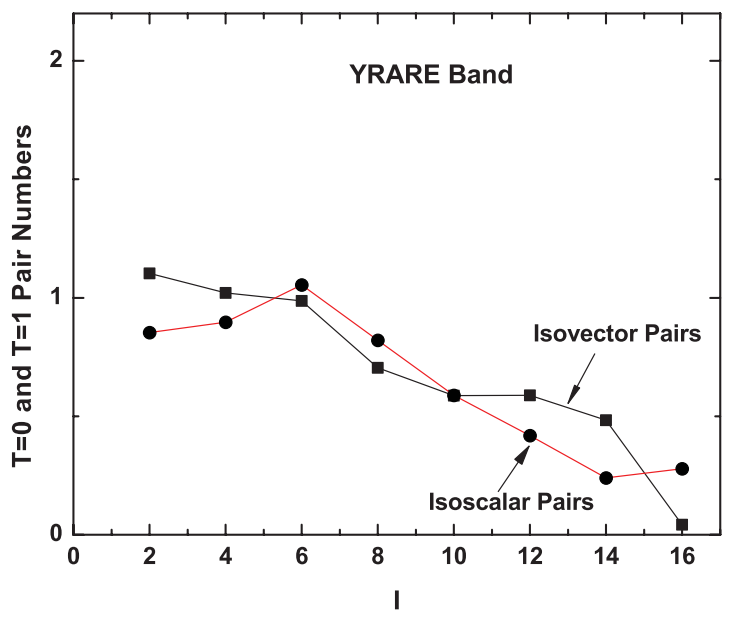

FIG. 18. (Color online) Calculated numbers of isovector $S^{\dagger}$ pairs and isoscalar $P^{\dagger}$ pairs in the first excited (yrare) band of ${ }^{48} \mathrm{Cr}$ for the optimal values of the Hamiltonian parameters.

probed the role of the various modes of pairing on deformation with and without a spin-orbit term. We did this as a function of the number of neutrons and protons, so as to assess the role both of a neutron excess and of the number of active particles.

Some of the conclusions that emerged are (1) in the absence of a spin-orbit term, isoscalar and isovector pairing have identical effects at $N=Z$, but isoscalar pairing ceases to have an appreciable effect for nuclei with just two excess neutrons; (2) the nonrotational character of ${ }^{44} \mathrm{Ti}$ cannot be explained solely in terms of spin-orbit effects, but requires pairing for its understanding; (3) in the presence of a spin-orbit interaction, isoscalar pairing is suppressed even at $N=Z$; (4) the fact that the ground state of ${ }^{46} \mathrm{~V}$ has $J^{\pi}=0^{+} T=1$ derives primarily from the spin-orbit interaction and its effect of suppressing isoscalar pairing; (5) the known backbend in ${ }^{48} \mathrm{Cr}$ has its origin in isovector pairing and does not derive from level crossing; and (6) in the region of the ${ }^{48} \mathrm{Cr}$ backbend, the numbers of isoscalar and isovector pairs behave quite differently in the yrast and yrare bands.

\section{ACKNOWLEDGMENTS}

The work reported herein began while two of the authors, S. P. and N. S., were visiting the Consejo Superior de Investigaciones Científicas in Madrid, whose hospitality is gratefully acknowledged. Much of it was carried out while Y. L. was visiting the Bartol Research Institute of the University of Delaware, whose hospitality is likewise acknowledged. The work of S. P., B. T., and Y. L. was supported in part by the National Science Foundation under Grant No. PHY-0854873, that of N.S. by the Romanian Ministry of Education and Research through CNCSIS Grant Idei No. 1975, that of A. P. by the Projects No. FPA2009-13377 MICINN(Spain) and No. HEPHACOS S2009/ESP-1473 Comunidad de Madrid(Spain), and that of Y. M. Z. and Y. L. by the National Science Foundation of China under Grant No. 10975096 and by the Chinese Major State Basic Research Developing Program under Grant No. 2007CB815000. 
[1] A. L. Goodman, Adv. Nucl. Phys. 11, 263 (1979).

[2] J. Dobeš and S. Pittel, Phys. Rev. C 57, 688 (1998).

[3] S. C. Pang, Nucl. Phys. A 128, 497 (1965); J. A. Evans, G. G. Dussel, E. E. Macqueda, and R. P. J. Perazzo, ibid. 367, 77 (1981); G. G. Dussel, E. E. Macqueda, R. P. J. Perazzo, and J. A. Evans, ibid. 450, 164 (1986).

[4] B. Errea, Ph.D. Thesis, Consejo Superior de Investigaciones Científicas, 2009.

[5] S. Lerma H., B. Errea, J. Dukelsky, and W. Satula, Phys. Rev. Lett. 99, 032501 (2007).
[6] N. Sandulescu, B. Errea, and J. Dukelsky, Phys. Rev. C 80, 044335 (2009).

[7] A. Poves and G. Martinez-Pinedo, Phys. Lett. B 430, 203 (1998).

[8] J. P. Elliott, Proc. R. Soc. London, Ser. A 245, 562 (1956).

[9] G. F. Bertsch and S. Baroni, arXiv:nucl-th/0904.2017v2.

[10] K. H. Bhatt and J. B. McGrory, Phys. Rev. C 3, 2293 (1971).

[11] A. P. Zuker, J. Retamosa, A. Poves, and E. Caurier, Phys. Rev. C 52, R1741 (1995). 